\title{
Preoperative Imaging in Colorectal Liver Metastases: Current Practices
}

\author{
Travis J. Smith · Elena Korngold · Susan L. Orloff
}

Published online: 25 December 2013

(C) Springer Science + Business Media New York 2013

\begin{abstract}
Surgical resection offers the only treatment associated with long-term survival for colorectal cancer (CRC) with hepatic metastasis. Imaging studies are paramount for the identification of potential surgical candidates. Synchronous hepatic disease is identified at presentation in 20-30\% of patients and ultimately, hepatic metastases occur in over $50 \%$ of patients diagnosed with CRC. Computed tomography (CT) has been the imaging standard to identify patients with hepatic metastasis, but the array of preoperative imaging has expanded over the past several years. Currently, ultrasound, CT, magnetic resonance imaging, and positron emission tomography all play a vital role in the management and treatment of stage IV CRC. In addition, patient characteristics such as steatosis and treatment such as neoadjuvant chemotherapy affect imaging of hepatic metastases. The goal of this review is to discuss the current standing of imaging modalities used in the perioperative care of patients with colorectal liver metastasis.
\end{abstract}

This article is part of the Topical Collection on Colorectal Liver Metastases.

\footnotetext{
T. J. Smith $(\bowtie)$

Department of General and Vascular Surgery, Gundersen Health

System, 1900 South Avenue, La Crosse, WI 54601, USA

e-mail: tjsmit1@gundersenhealth.org

E. Korngold

Department of Radiology, Oregon Health and Science

University, 3181 S.W. Sam Jackson Park Rd, Portland,

OR 97239, USA

S. L. Orloff $(\square)$

Department of Surgery, Oregon Health and Science University,

3181 S.W. Sam Jackson Park Rd, Portland, OR 97239, USA

e-mail: orloffs@ohsu.edu
}

Keywords Colorectal liver metastases - Preoperative imaging · Computed tomography · Ultrasound · Magnetic resonance imaging (MRI) - Positron emission tomography

\section{Introduction}

Colorectal cancer (CRC) is the second leading cause of cancer-related death in the western world. Following regional lymph node involvement, the liver is the most frequent site of distant metastasis, with $20-30 \%$ of patients presenting with synchronous hepatic metastasis on initial staging $[1,2]$. Ultimately, metachronous hepatic metastases occur in over $50 \%$ of patients diagnosed with CRC [3, 4]. Evaluation of these complex patients requires a thorough history and physical examination, laboratory evaluation, and imaging studies. Imaging studies are paramount to preoperative staging and identification of potential surgical candidates. Surgical resection offers the only treatment associated with long-term survival for CRC with hepatic metastasis [5]. In compliance with National Comprehensive Cancer Network Guidelines, computed tomography (CT) has been used as the standard of care to identify patients with hepatic metastasis [6, 7]. Many updates in the field of imaging have occurred in the last 5 years. Currently, ultrasound (US), CT, magnetic resonance imaging (MRI), positron emission tomography (PET), and intraoperative imaging modalities collectively play a vital role in the management and treatment of stage IV CRC. In addition, patient characteristics such as steatosis and treatment options such as neoadjuvant chemotherapy therapy affect imaging of hepatic metastases. The goal of this review is to discuss the current practices in imaging modalities used in the perioperative care of patients with colorectal liver metastasis. 


\section{Methods}

A systematic review of the literature from January 2003 to July 2013 was performed using the following terms: "colon neoplasm" or "rectal neoplasms;" and "liver neoplasms;" and "MRI" or "MR" or "CT" or "SCT" or "MSCT" or "MDCT" or "CTAP" or "PET" or "US" or "IOUS" or "diagnostic tests". For each term, the "explode" option was used. Additional limits of human subjects and the English language were applied and 466 articles were initially identified.

Reviewers scanned the title, abstract, and key words of the retrieved records and obtained full text of the relevant articles. Reference lists were then searched to identify additional articles. In order to focus on the most current literature, relevant articles from the past 5 years were preferentially selected.

\section{Results}

\section{Ultrasound}

While traditional US has limited use in preoperative imaging of colorectal hepatic metastasis, several advances in the field of contrast-enhanced US and intraoperative US show promising results.

Traditional US has an advantage of providing very costeffective and widely available imaging (Fig. 1). Ultrasound provides a pooled sensitivity and specificity 63.0 and $97.6 \%$ [8••]. While contrast-enhanced US (CEUS) is not available in the United States outside of echocardiography, it deserves mention. Recent prospective studies comparing noncontrast-enhanced US, CT, and MRI to transcutaneous contrast-enhanced US have demonstrated increased sensitivity of contrast-enhanced US to 80.0-91.2\% [9-11]. This sensitivity range is comparable to multidetector CT (MDCT). Another prospective study that directly compared CEUS to MDCT demonstrated that MDCT had a higher sensitivity for detection of hepatic metastases but this was not statistically significant (89 vs. $80 \% ; P=0.06$ ) [12]. While these are promising results and may provide an inexpensive imaging alternative in the future, CEUS in the United States is dependent on Federal Drug Administration approval of the contrast agent. Ultrasound also has the notable weakness of being operator-dependent.

Intraoperative US also warrants brief discussion. Intraoperative US (IOUS) has historically had a significant impact on intraoperative decision-making for the management of CRC with liver metastases. With improved axial imaging, the impact of IOUS has decreased and its performance has been questioned [13, 14]. Despite this, in a recent series of patients who underwent preoperative

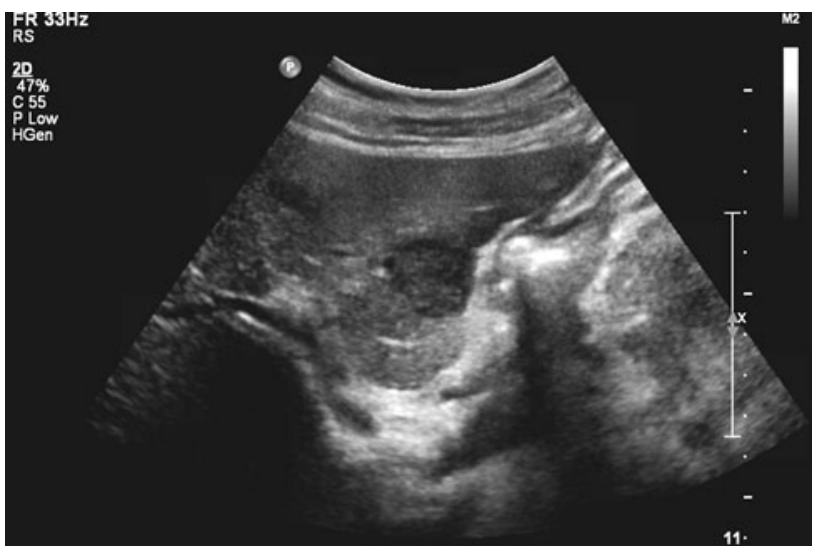

Fig. 1 Transabdominal ultrasound using a 5-MHz curved transducer demonstrates round hypoechoic (darker) lesion in the left hepatic lobe

MDCT, IOUS reported the identification of additional metastases in $16 \%$ and a resultant change in clinical management in $9 \%$ [15•]. Contrast-enhanced IOUS has also shown potential with improved sensitivity and specificity compared to traditional IOUS, even in the setting of neoadjuvant therapy $[16,17 \bullet \cdot]$. In one prospective study, consecutive patients underwent preoperative MDCT, IOUS, and contrast-enhanced IOUS. The results indicated that contrast-enhanced IOUS altered the intraoperative decision-making in $20 \%$ of patients. Two lesions that were classified as indeterminate by preoperative MDCT and IOUS were classified as benign, based on contrastenhanced IOUS. Two additional hepatic metastatic CRC lesions were identified by contrast-enhanced IOUS: one lesion that had been classified as indeterminate, and another lesion was not previously identified.

Today, transabdominal US has a very limited role in the evaluation of patients with metastatic colorectal cancer to the liver, but, given time, contrast-enhanced US may play an emerging role. Intraoperative US remains a valuable tool for intraoperative decision-making.

\section{Computed Tomography}

Multidetector row computed tomography (MDCT) remains the mainstay for staging colorectal liver metastases (Fig. 2). MDCT is a reliable tool with pooled sensitivity and specificity of 74.8 and $95.6 \%$, ranging from 48.4 to 100 and 80 to $100 \%$ (Table 1) [8••, 18•]. When limited to only prospective studies on a per patient basis, sensitivity and specificity are 83.3 and $95.7 \%$, respectively. As such, CT of the chest, abdomen, and pelvis with IV contrast is the recommended initial imaging modality in staging both colon and rectal cancer by current National Comprehensive Cancer Network (NCCN) guidelines [6, 7].

Advantages of $\mathrm{CT}$ include its easy availability and speed. Disadvantages of CT include the risk of ionizing 


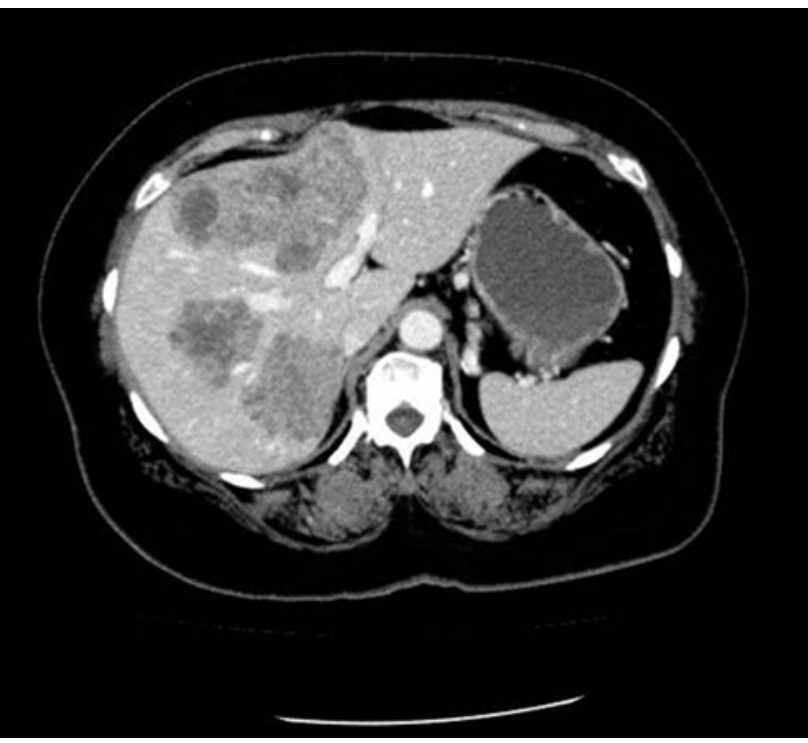

Fig. 2 Routine contrast-enhanced CT of the abdomen in the portal venous phase (70-80 s after iodinated IV contrast injection) demonstrates heterogeneous, predominately hypodense (dark) metastases within segment four and the right hepatic lobe

Table 1 Sensitivity and specificity estimates for each imaging modality

\begin{tabular}{llll}
\hline Imaging & Per lesion basis & Per patient basis \\
\cline { 3 - 4 } & $\begin{array}{l}\text { Mean } \\
\text { sensitivity (\%) }\end{array}$ & $\begin{array}{l}\text { Mean } \\
\text { sensitivity }(\%)\end{array}$ & $\begin{array}{l}\text { Mean } \\
\text { specificity (\%) }\end{array}$ \\
\hline CT & 74.4 & 83.6 & 94.9 \\
MR & 80.3 & 88.2 & 92.5 \\
FDG PET & 81.4 & 94.1 & 95.7 \\
FDG PET/ & 66.2 & 96.5 & 97.2 \\
CT & & &
\end{tabular}

Adapted from Niekel et al. [18•]; copyright 2010, Radiological Society of North America; with permission

$C T$ computed tomography, $M R$ magnetic resonance, FDG PET fluorodeoxyglucose positron emission tomography

radiation. While this is a concern, the population of patients with CRC is generally older and less susceptible to the long-term risk associated with ionizing radiation. Contrast agents also have the risk of nephrotoxicity. The greatest limitation of CT is its inability to characterize sub-centimeter lesions.

At this time, MDCT is a convenient and reliable imaging modality that should be used as the imaging staging modality of choice in most patients with the diagnosis of CRC. Special circumstances in which other modalities should be considered include patients with renal impairment or young age and risk of radiation exposure.

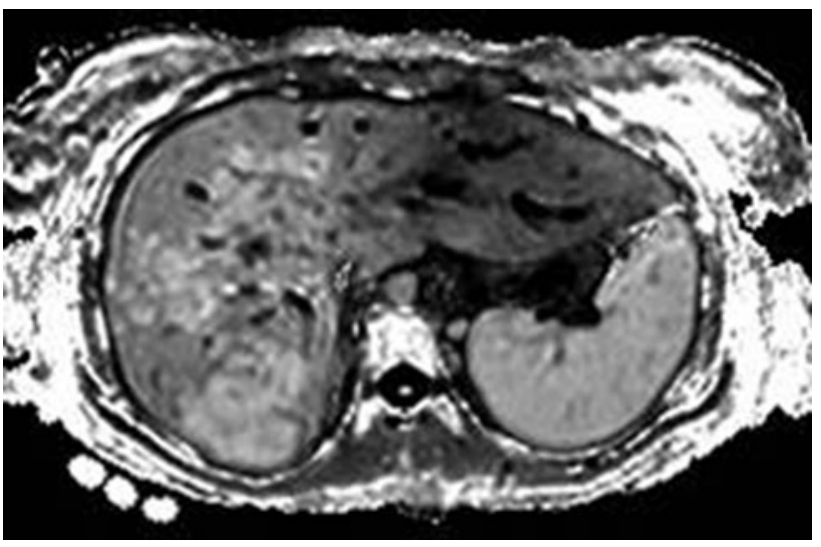

Fig. 3 Magnetic resonance diffusion-weighted imaging illustrating patchy areas of increased (brighter) signal within segment four and the right lobe indicate restricted diffusion from metastases

\section{Magnetic Resonance Imaging}

Over the past 5 years, magnetic resonance imaging (MRI) has evolved. New contrast agents, as well as MRI software and hardware, have resulted in the advancement of this non-invasive imaging. The different modalities, series, and contrast agents may be initially daunting; however, in collaboration with our radiology colleagues, this imaging modality can be more easily understood and applied to our patients.

\section{MR Review}

1. Magnet: Strength measured in Tesla (T). The stronger the magnet, the higher the spatial resolution.

2. Spin echo (SE): Standard anatomic MR imaging.

3. Echo planar imaging (EPI): Add on to SE for diffusion or perfusion sequences.

4. Diffusion-weighted imaging (DWI): Ability of MR to assess the diffusion of water in tissue. Restricted diffusion occurs when water has decreased ability to diffuse through tissue that is seen with increase cellularity or fibrosis and results in a bright signal on DWI. Restricted diffusion is seen in tumors, edema, and fibrosis. DWI can be used with or without contrast agents (Fig. 3).

\section{Contrast Agents}

1. Gadolinium: Standard MR contrast agent which is gadopentetate dimeglumine (Gd-DTPA) - Magnevist $^{\circledR}$ (Bayer HealthCare Pharmaceuticals, Wayne, NJ, USA). CRC metastases generally display hypovascular enhancement patterns (Fig. 4).

2. Hepatobiliary agents 


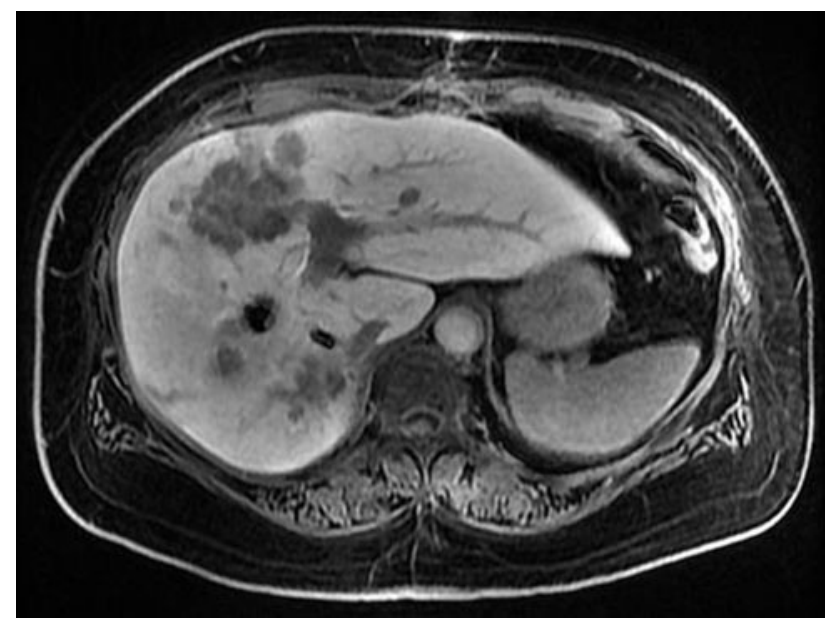

Fig. 4 Non-contrast T1-weighted fat-saturated image of the liver demonstrating hypointense (darker gray) metastases. Very dark focus in the right lobe is an artifact from embolization coil material
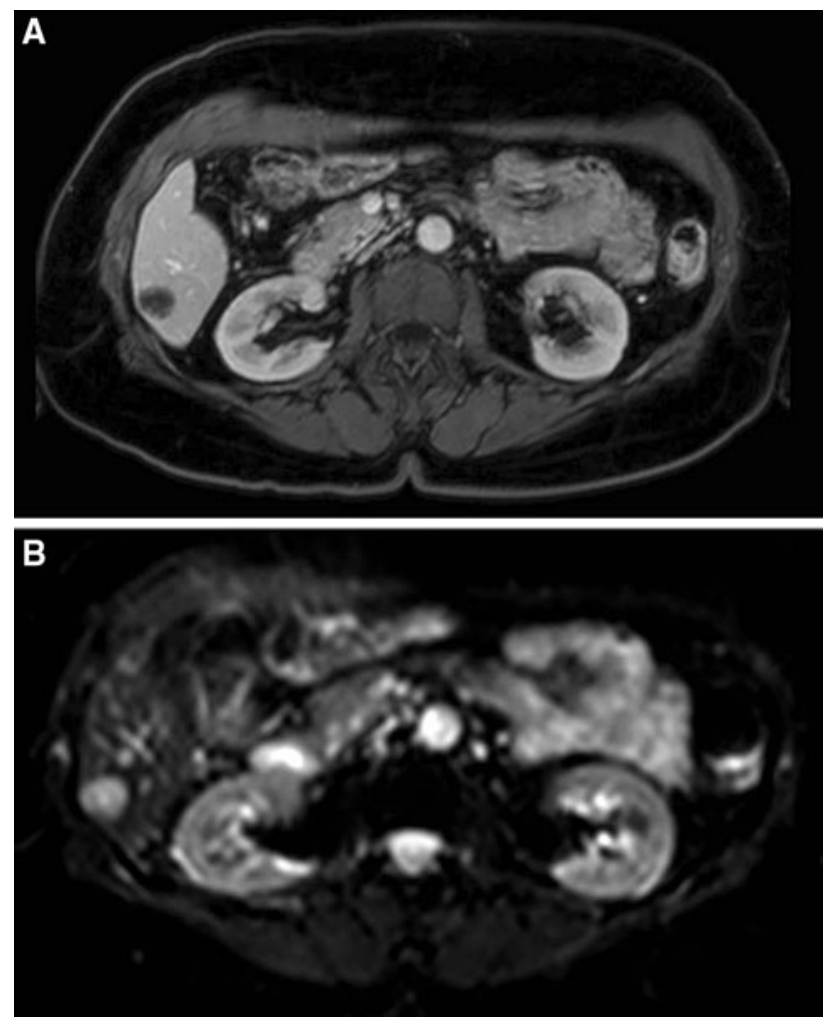

Fig. 5 Magnetic resonance imaging with Eovist $^{\circledR}$ and diffusionweighted imaging illustrating a hypointense metastasis in segment six (a), with corresponding restricted diffusion (b)

a. Gadolinium ethoxybenzyl diethylenetriamine penta-acetic acid (Gd-EOB-DTPA) - Eovist ${ }^{\circledR}$ (Bayer HealthCare Pharmaceuticals): This agent is taken up in functional hepatocytes and excreted into the biliary system. Delayed imaging shows retained uptake in normal liver, but enhanced washout in non-hepatocyte-containing lesions that result in increasing the identification of hepatic metastases (Fig. 5a).

b. Super Paramagnetic iron oxide (SPIO)-Feridex ${ }^{\circledR}$ (Bayer HealthCare Pharmaceuticals): SPIO are nano-sized particles that are sequestered by $\mathrm{Ku}$ pffer cells resulting in darkening of the normal liver parenchyma. Normal liver becomes dark on T2 images and hepatic metastases stand out.

c. Mangafodipir dipyridoxyl diphosphate (MnDPDP)—Teslascan ${ }^{\circledR}$ (Amersham Health, Princeton, NJ, USA): Hepatocyte-specific contrast agent, which is taken up by hepatocytes more than by abnormal or cancerous tissue, making the normal hepatic parenchyma appear brighter then metastasis on $\mathrm{T} 1$ imaging.

Additional software capabilities also offer three-dimensional reconstructions. MR with Gd-EOB-DTPA, the most common hepatic contrast agent, results in improved sensitivity and specificity for detecting CRC with liver metastasis [19]. Early studies that compared different MR modalities directly, specifically Gadolinium and SPIO, identified no difference in accuracy. When these modalities are compared to $\mathrm{CT}$, both MR techniques provide superior accuracy that is more sensitive for lesions $<1 \mathrm{~cm}$ in size [20]. As experience with hepatic-specific contrast agents evolves, these agents result in improving lesion identification. A recent prospective study of patients who underwent SPIO MR and Gadobenate (GbD) MR identified $100 \%$ sensitivity in the SPIO group compared to $91 \%$ in the $\mathrm{GbD}$ group [21]. Both MR techniques had higher sensitivity when compared to $82 \%$ with CT.

EOB-DTPA improves lesion detection, and DWI assists with lesion characterization. These two modalities in combination result in improved sensitivity and accuracy of small colorectal liver metastases (Fig. 5b) versus either modality independently $[22,23]$. These combined imaging modalities ultimately translate into improved detection of CRCLM, specifically for small lesions $(<1 \mathrm{~cm})$. While SPIO and Mn-DPDP also improve the sensitivity of MR, these agents are not widely used [24-26]. Pooled sensitivity and specificity analysis for MR imaging were 81.1 and $97.2 \%$, ranging from 64.3 to 100 and 90.6 to $98.4 \%$ [8••]. When assessing prospective trials on a per patient basis, the sensitivity and specificity of MR is 88.2 and $92.5 \%$, respectively. Table 2 demonstrates estimated sensitivities of CT, MR, and MR subgroups.

Magnetic resonance imaging has three advantages over the other imaging modalities discussed. The first advantage is lack of ionizing radiation. A more significant advantage is the recent development and subsequent accuracy of 
Table 2 Sensitivity estimate for subgroups on a per lesion basis

\begin{tabular}{llc}
\hline Subgroup & \multicolumn{2}{l}{ Mean sensitivity $(\%)$} \\
\cline { 2 - 3 } & MR & $\mathrm{CT}$ \\
\hline Lesion size & & \\
$<1 \mathrm{~cm}$ & 60.2 & 47.3 \\
$\geq 1 \mathrm{~cm}$ & 89.0 & 86.7 \\
Study year & & \\
$\quad$ Prior to January 2004 & 70.2 & 73.4 \\
After January 2004 & 84.9 & 74.9 \\
MR technique & & \\
$\quad$ Unenhanced & 78.2 & $\mathrm{NA}$ \\
Contrast-enhanced & & $\mathrm{NA}$ \\
$\quad$ Mangafodipir trisodium & 86.0 & \\
$\quad$ SPIO & 79.5 & \\
$\quad$ Gadoterate or gadopentetate & 79.8 & \\
\hline
\end{tabular}

Adapted from Niekel et al. [18•]; copyright 2010, Radiological Society of North America; with permission

$M R$ magnetic resonance, $C T$ computed tomography, SPIO superparamagnetic iron oxide, $N A$ not applicable

hepatic-specific contrast agents. While these contrast agents continue to have some risk of nephrotoxicity in the form of nephrogenic fibrosis, this entity only occurs in patients with severe baseline renal impairment. The greatest strength of MRI is its ability to characterize subcentimeter lesions, which is a significant drawback in CT imaging modalities. Disadvantages of MRI are four-fold: (1) cost, (2) availability, (3) exam duration, and (4) operator expertise. While none of these obstacles are insurmountable, as healthcare becomes more cost conscious, the use of MR will have to be tailored appropriately.

Currently, MRI should be used in the preoperative evaluation of patients with CRC with known or suspected hepatic metastasis that cannot be completely characterized by other imaging modalities.

\section{Positron Emission Tomography}

Positron emission tomography (PET) provides a metabolic map of glucose uptake of the entire body, resulting in identification of metabolic activity in potential lesions. This modality continues to play a major role in the management of patients with stage IV CRC (Fig. 6). It has an overall sensitivity of $75.9-100 \%$ and specificity of 96.0-100 \% in detecting hepatic metastasis [8••, 27, 28• 29]. The sensitivity of PET declines with decreasing lesion size. For lesions $>2 \mathrm{~cm}$, PET/CT has a sensitivity of $100 \%$ [30]. Sensitivity decreases to 54 and $32 \%$ for lesions $1-2$ and $<1 \mathrm{~cm}$ in size, respectively [30].

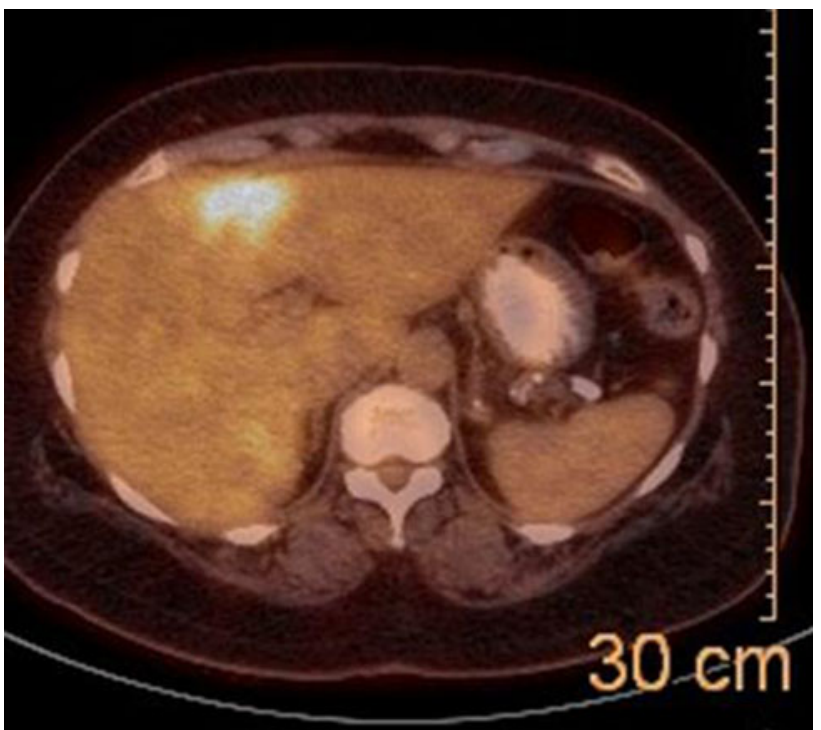

Fig. 6 Fused image of 18F-FDG-PET and co-registered non-contrast CT demonstrating a focus of increased FDG uptake (bright compared to background) in segment four, corresponding to a hepatic metastasis

PET offers the added benefit of identification of extrahepatic metastasis with reported sensitivities up to $100 \%$ [31]. Compared to CT, PET is superior for the detection of extrahepatic metastases [29, 32]. In a prospective trial, PET sensitivity was equivalent to CT for detection of hepatic metastasis but was superior for extrahepatic sites (63 vs. $25 \%$ ) [32]. In this study, PET identified 5 additional patients with extrahepatic disease but also falsely upstaged 3 patients out of a total of 59 [32]. Due to PET's relatively high sensitivity, specificity and ability to identify extrahepatic disease, PET imaging results in clinically significant findings that lead to changes in management in $9-32 \%$ of patients with CRC [31-33]. PET also plays an important role in the detection of postoperative systemic and local recurrence [29].

Disadvantages of PET must also be considered. PET/CT results in exposure to ionizing radiation. In addition, the metabolic activity is not specific to metastatic disease and may result in false positive studies.

Currently, routine use of PET is not recommended for diagnosis or staging of clinical stage I-III CRC; however, it is recommended in the following situations:

1. Preoperative assessment of CRC liver metastasis patients prior to surgical resection

2. Determining management if conventional imaging is equivocal for the presence of metastatic disease

3. Determining site of recurrence in the setting of rising CEA when conventional evaluation fails to identify metastatic disease. 


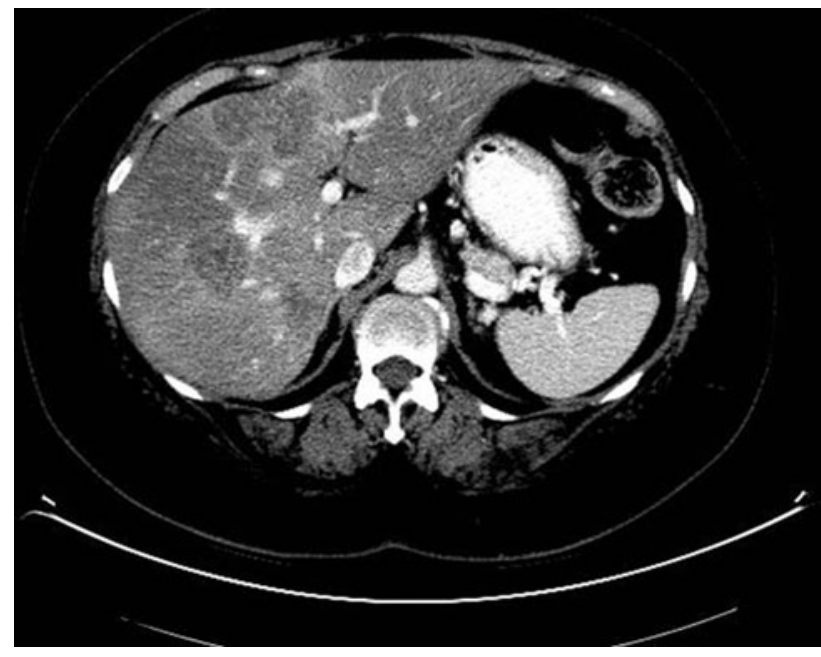

Fig. 7 Computed tomography (CT) scan of the same patient as depicted in Fig. 2 after chemotherapy, demonstrating diffuse hepatic steatosis, indicated by lower density of the liver when compared to the pre-treatment image. The metastases are smaller but also much harder to see due to overall decrease in liver density, which is now similar to the density of the metastases. MRI becomes the modality of choice in this scenario, when metastatic margins become harder to measure and new metastases could be missed on CT

\section{Special Situations}

\section{Neoadjuvant Chemotherapy}

Patients with untreated hepatic metastases from CRC have a negligible 5-year survival [34], while patients treated with combination chemotherapy and surgical resection have 5-year survival rates approaching $60 \%[5,35,36]$. With the use of neoadjuvant chemotherapy, the population of patients with CRC and hepatic metastasis eligible for surgery continues to expand [37]. Neoadjuvant regimens including irinotecan and oxliplatin have the most biologic activity but also result in hepatotoxicity [38-40]. Neoadjuvant therapy results in decreased sensitivity of preoperative imaging for identification of existing colorectal hepatic metastasis [41, 42]. As the use of neoadjuvant therapy expands, preoperative decisions regarding eligible surgical candidates will become more challenging. Accurate detection and localization of all metastatic disease is essential for determining which patients have the potential to undergo a curative $\mathrm{R} 0$ resection.

Superparamagnetic iron oxide has been shown to be very sensitive for detecting sub-centimeter colorectal hepatic metastases [20]. A recent prospective study evaluating the sensitivity of SPIO-MRI using early contrastenhancing T1 imaging, which results in the creation of isointense liver/vasculature background in which liver metastases stand out, resulted in a detection rate of $77 \%$ in patients receiving neoadjuvant therapy versus $87 \%$ in patients who did not receive chemotherapy [26]. An additional study on SPIO-enhanced MRI following neoadjuvant chemotherapy for hepatic metastasis had a sensitivity of 92 versus $52 \%$ for PET/CT [43].

MR enhanced with both diffusion-weighted imaging and GD-EOB-DTPA contrast has also shown improved sensitivity in post-neoadjuvant therapy. In a retrospective study of 32 consecutive patients undergoing neoadjuvant chemotherapy with either DWI, GD-EOB-DTPA, or combined DWI and GD-EOB-DTPA MRI performed, the overall sensitivity was 60,74 , and $91 \%$, respectively, for detecting CRC liver metastasis post-chemotherapy [44••]. The combination of hepatic-specific contrast agents and DWI in MRI has resulted in increased sensitivity and detection of hepatic metastasis post-neoadjuvant therapy for metastatic CRC. Prior to an attempted R0 surgery, the aforementioned modalities should be strongly considered in evaluating high-risk operative candidates who are classified as unresectable on initial CT staging, and those who have shown a significant imaging response on subsequent imaging.

Today, MR in combination with DWI and liver-specific contrast agents provide the most sensitive tool for detecting liver metastasis in CRC patients who have undergone neoadjuvant chemotherapy.

\section{Hepatic Steatosis}

Metabolic syndrome and subsequent non-alcoholic liver disease and non-alcoholic steatohepatitis are the most common causes of liver disease in the United States, and their incidence is increasing [45]. Chemotherapy can also result in steatosis of the liver $[39,46]$. The ability of noninvasive imaging studies to evaluate steatotic livers continues to evolve [47, 48•]. Hepatic steatosis that is secondary to patients' underlying medical comorbidities or due to neoadjuvant chemotherapy results in decreased density of the hepatic parenchyma making the detection of hypovascular colorectal liver metastases more difficult (Fig. 7). A recent retrospective analysis of 20 consecutive patients with CRC and hepatic metastases who had biopsyproven hepatic steatosis $(>25 \%)$ assessed the ability of MDCT and MRI to evaluate hepatic metastasis. MDCT identified $33(65 \%)$ and MRI identified 45 (88\%) lesions out of the total of 51 lesions identified pathologically [49]. MRI with Gd EOB GDTP was superior to 64 row MDCT scanners in detecting hepatic metastasis in the setting of a steatotic liver [49]. A similar study compared MDCT, MRI, and IOUS and confirmed the finding that MRI was superior to $\mathrm{CT}$ in detecting $<1 \mathrm{~cm}$ CRC hepatic metastases in a steatotic liver (97 vs. $72 \%$ ) [50]. The MRI protocol utilized a 3T MRI with Gd-EOB-DTPA, a hepatic-specific contrast agent. The CT was a multidetector 64-row scanner. Intraoperative US identified all lesions but resulted in two false positive lesions that were not confirmed on final 
pathology [50]. The effect of steatosis on echogenicity of colorectal hepatic metastasis on IOUS was also evaluated prospectively in 126 patients [51••]. The majority of CRC with liver metastases were hypoechoic $(48 \%)$, followed closely by isoechoic (34\%), while only $18 \%$ were hyperechoic. In steatotic livers, IOUS imaging quality was worse, and CRC with liver metastases were significantly more likely to be hypoechoic, which actually made the lesions more conspicuous [51••]. While MRI is superior to other preoperative imaging modalities identifying CRC with liver metastases in fatty livers, IOUS continues to perform well.

In the setting of hepatic steatosis, MR with hepaticspecific contrast agents is superior to MDCT in detecting CRC with liver metastases. Intraoperative US imaging quality is diminished; however, it still allows for visualization of pertinent lesions and IOUS remains an important adjunct for intraoperative decision-making.

\section{Conclusions}

Hepatic metastectomy offers the only treatment associated with long-term survival for CRC with hepatic metastasis. Unfortunately, hepatic metastases occur in over $50 \%$ of patients diagnosed with CRC, and the majority of these patients present with unresectable disease. Imaging studies are paramount not only for identification of hepatic metastasis but also for characterization of these lesions to identify potential surgical candidates. Today, intraoperative US remains a valuable tool for intraoperative decisionmaking. Contrast-enhanced US may play an emerging role in the future. Computed tomography is a convenient reliable imaging modality that is the staging modality of choice in most patients with the diagnosis of CRC. Magnetic resonance imaging technology has had significant advancement and should be used in the preoperative evaluation of patients with CRC with known or suspected hepatic metastasis that cannot be completely characterized by other imaging modalities. The greatest advantage of MRI includes identification and characterization of small lesions. In addition, it provides the greatest accuracy in patients who have received neoadjuvant chemotherapy or in those with hepatic steatosis. While routine use of PET is not recommended for diagnosis or staging in early stage $\mathrm{CRC}$, it is recommended in the preoperative assessment of CRC liver metastasis prior to surgical resection. Innovations in preoperative imaging continue to evolve, resulting in improved identification and characterization of CRC hepatic metastasis. The use of MRI and the recent attendant liver contrast-enhanced agents will allow for more appropriate patient selection for surgical resection with the goal of long-term survival with liver resection.

\section{Compliance with Ethics Guidelines}

Conflict of Interest Susan L. Orloff has received funding from the National Institutes of Health, has served as an AHPBA councilor and foundation board member, as an IHPBA research committee member, and as an American Association of Liver Diseases councilor. Travis J. Smith and Elena Korngold declare that they have no conflict of interest.

Human and Animal Rights and Informed Consent This article does not contain any studies with human or animal subjects performed by any of the authors.

\section{References}

Papers of particular interest, published recently, have been highlighted as:

- Of importance

•- Of major importance

1. Muratore A, Zorzi D, Bouzari H, et al. Asymptomatic colorectal cancer with un-resectable liver metastases: immediate colorectal resection or up-front systemic chemotherapy? Ann Surg Oncol. 2007; $14: 766-70$.

2. Hayashi M, Inoue $\mathrm{Y}$, Komeda $\mathrm{K}$, et al. Clinicopathological analysis of recurrence patterns and prognostic factors for survival after hepatectomy for colorectal liver metastasis. BMC Surg. 2010;10:27.

3. Van Cutsem E, Nordlinger B, Adam R, European Colorectal Metastases Treatment Group, et al. Towards a pan-European consensus on the treatment of patients with colorectal liver metastases. Eur J Cancer. 2006;42:2212-21.

4. Manfredi S, Lepage C, Hatem C, et al. Epidemiology and management of liver metastases from colorectal cancer. Ann Surg. 2006;244:254-9.

5. Kopetz S, Chang GJ, Overman MJ, et al. Improved survival in metastatic colorectal cancer is associated with adoption of hepatic resection and improved chemotherapy. J Clin Oncol. 2009;27: 3677-83.

6. National Comprehensive Cancer Network. NCCN Clinical Practice Guidelines in Oncology (NCCN Guidelines ${ }^{\circledR}$ ). Colon Cancer. Version I. 2014. http://www.nccn.org/professionals/ physician_gls/f_guidelines.asp\#colon. Accessed 16 Oct 2013.

7. National Comprehensive Cancer Network. NCCN Clinical Practice Guidelines in Oncology (NCCN Guidelines $\left.{ }^{\circledR}\right)$. Rectal Cancer. Version I. 2014. http://www.nccn.org/professionals/ physician_gls/f_guidelines.asp\#rectal. Accessed 16 Oct 2013.

8. - Floriani I, Torri V, Rulli E, et al. Performance of imaging modalities in diagnosis of liver metastases from colorectal cancer: a systematic review and meta-analysis. J Magn Reson Imaging. 2010;31:19-31. This systematic review objectively compared multiple modern imaging modalities in patients with a diagnosis of colorectal cancer liver metastases.

9. Konopke R, Kersting S, Bergert $\mathrm{H}$, et al. Contrast-enhanced ultrasonography to detect liver metastases: a prospective trial to compare transcutaneous unenhanced and contrast-enhanced ultrasonography in patients undergoing laparotomy. Int J Colorectal Dis. 2007;22:201-7.

10. Larsen LP, Rosenkilde M, Christensen H, et al. The value of contrast enhanced ultrasonography in detection of liver metastases from colorectal cancer: a prospective double-blinded study. Eur J Radiol. 2007;62:302-7. 
11. Dietrich CF, Kratzer W, Strobe D, et al. Assessment of metastatic liver disease in patients with primary extrahepatic tumors by contrast-enhanced sonography versus CT and MRI. World J Gastroenterol. 2006;12:1699-705.

12. Larsen LP, Rosenkilde M, Christensen H, et al. Can contrastenhanced ultrasonography replace multidetector-computed tomography in the detection of liver metastases from colorectal cancer? Eur J Radiol. 2009;69:308-13.

13. Tamandl D, Herberger B, Gruenberger B, et al. Adequate preoperative staging rarely leads to a change of intraoperative strategy in patients undergoing surgery for colorectal cancer liver metastases. Surgery. 2008;143:648-57.

14. Sahani DV, Kalva SP, Tanabe KK, et al. Intraoperative US in patients undergoing surgery for liver neoplasms: comparison with MR imaging. Radiology. 2004;232:810-4.

15. • van Vledder MG, Pawlik TM, Munireddy S, et al. Factors determining the sensitivity of intraoperative ultrasonography in detecting colorectal liver metastases in the modern era. Ann Surg Oncol. 2010;17:2756-63. Evaluates IOUS in era of modern imaging.

16. Takahashi M, Hasegawa K, Arita J, et al. Contrast-enhanced intraoperative ultrasonography using perfluorobutane microbubbles for the enumeration of colorectal liver metastases. Br J Surg. 2012;99:1271-7.

17. $\bullet$ Ruzzenente A, Conci S, Iacono C, et al. Usefulness of contrast-enhanced intraoperative ultrasonography (CE-IOUS) in patients with colorectal liver metastases after preoperative chemotherapy. J Gastrointest Surg. 2013;17:281-87. This article supports the use of IOUS in the setting of modern axial imaging.

18. - Niekel MC, Bipat S, Stoker J. Diagnostic imaging of colorectal liver metastases with CT, MR imaging, FDG PET, and/or FDG PET/CT: a meta-analysis of prospective studies including patients who have not previously undergone treatment. Radiology. 2010; 257:674-84. This article supports the use of IOUS in the setting of modern axial imaging.

19. Bluemke DA, Sahani D, Amendola M, et al. Efficacy and safety of MR imaging with liver-specific contrast agent: U.S. multicenter phase III study. Radiology. 2005;237:89-98.

20. Ward J, Robinson PJ, Guthrie JA, et al. Liver metastases in candidates for hepatic resection: comparison of helical CT and gadolinium- and SPIO-enhanced MR imaging. Radiology. 2005; 237:170-80.

21. Hekimoglu K, Ustundag Y, Dusak A, et al. Small colorectal liver metastases: detection with SPIO-enhanced MRI in comparison with gadobenate dimeglumine-enhanced MRI and CT imaging. Eur J Radiol. 2011;77:468-72.

22. Löwenthal D, Zeile M, Lim WY, et al. Detection and characterization of focal liver lesions in colorectal carcinoma patients: comparison of diffusion-weighted and Gd-EOB-DTPA enhanced MR imaging. Eur Radiol. 2011;21:832-40.

23. Chung WS, Kim MJ, Chung YE, et al. Comparison of gadoxetic acid-enhanced dynamic imaging and diffusion-weighted imaging for the preoperative evaluation of colorectal liver metastases. J Magn Reson Imaging. 2011;34:345-53.

24. Ba-Ssalamah A, Uffmann M, Saini S, et al. Clinical value of MRI liver-specific contrast agents: a tailored examination for a confident non-invasive diagnosis of focal liver lesions. Eur Radiol. 2009;19:342-57.

25. King LJ, Burkill GJ, Scurr ED, et al. MnDPDP enhanced magnetic resonance imaging of focal liver lesions. Clin Radiol. 2002;57:1047-57.

26. Smith JT, Ward J, Guthrie JA, et al. Detection of colorectal metastases in patients being treated with chemotherapy utilising SPIO-MRI: a radiological-pathological study. Magn Reson Imaging. 2012;30:1446-53.
27. Bipat S, van Leeuwen MS, Comans EF, et al. Colorectal liver metastases: CT, MR imaging, and PET for diagnosis-meta-analysis. Radiology. 2005;237:123-31.

28. - Chan K, Welch S, Walker-Dilks C, Raifu A. Ontario provincial Gastrointestinal Disease Site Group. Evidence-based guideline recommendations on the use of positron emission tomography imaging in colorectal cancer. Clin Oncol (R Coll Radiol). 2012;24:232-49. Current guidelines for use of PET in CRC.

29. Chua SC, Groves AM, Kayani I, et al. The impact of 18F-FDG PET/CT in patients with liver metastases. Eur J Nucl Med Mol Imaging. 2007;34:1906-14.

30. Coenegrachts K, De Geeter F, ter Beek L, et al. Comparison of MRI (including SS SE-EPI and SPIO-enhanced MRI) and FDGPET/CT for the detection of colorectal liver metastases. Eur Radiol. 2009;19:370-9.

31. Huguet EL, Old S, Praseedom RK, et al. F18-FDG-PET evaluation of patients for resection of colorectal liver metastases. Hepatogastroenterology. 2007;54:1667-71.

32. Truant S, Huglo D, Hebbar M, et al. Prospective evaluation of the impact of [18F]fluoro-2-deoxy-D-glucose positron emission tomography of resectable colorectal liver metastases. Br J Surg. 2005;92:362-9.

33. Facey K, Bradbury I, Laking G, Payne E. Overview of the clinical effectiveness of positron emission tomography imaging in selected cancers. Health Technol Assess. 2007;11:iii-iv, xi-267.

34. Jonker DJ, Maroun JA, Kocha W. Survival benefit of chemotherapy in metastatic colorectal cancer: a meta-analysis of randomized controlled trials. Br J Cancer. 2000;82:1789-94.

35. Fernandez FG, Drebin JA, Linehan DC, et al. Five-year survival after resection of hepatic metastases from colorectal cancer in patients screened by positron emission tomography with F-18 fluorodeoxyglucose (FDG-PET). Ann Surg. 2004;240:438-47.

36. Choti MA, Sitzmann JV, Tiburi MF, et al. Trends in long-term survival following liver resection for hepatic colorectal metastases. Ann Surg. 2002;235:759-66.

37. Adam R, Delvart V, Pascal G, et al. Rescue surgery for unresectable colorectal liver metastases downstaged by chemotherapy: a model to predict long-term survival. Ann Surg. 2004; 240:644-57.

38. Lee WM. Drug-induced hepatotoxicity. N Engl J Med. 1995;333: 1118-27.

39. Vauthey JN, Pawlik TM, Ribero D, et al. Chemotherapy regimen predicts steatohepatitis and an increase in 90-day mortality after surgery for hepatic colorectal metastases. J Clin Oncol. 2006;24:2065-72.

40. Karoui M, Penna C, Amin-Hashem M, et al. Influence of preoperative chemotherapy on the risk of major hepatectomy for colorectal liver metastases. Ann Surg. 2006;243:1-7.

41. Lubezky N, Metser U, Geva R, et al. The role and limitations of 18-fluoro-2-deoxy-D-glucose positron emission tomography (FDG-PET) scan and computerized tomography (CT) in restaging patients with hepatic colorectal metastases following neoadjuvant chemotherapy: comparison with operative and pathological findings. J Gastrointest Surg. 2007;11:472-8.

42. Robinson PJ. The effects of cancer chemotherapy on liver imaging. Eur Radiol. 2009;19:1752-62.

43. Bacigalupo L, Aufort S, Eberlé MC, et al. Assessment of liver metastases from colorectal adenocarcinoma following chemotherapy: SPIO-MRI versus FDG-PET/CT. Radiol Med. 2010;115:1087-100.

44. •• Macera A, Lario C, Petracchini M, et al. Staging of colorectal liver metastases after preoperative chemotherapy. Diffusionweighted imaging in combination with Gd-EOB-DTPA MRI sequences increases sensitivity and diagnostic accuracy. Eur Radiol. 2013;23:739-47. This report proves the use of MR with hepatic contrast and DWI to identify CRLM, even in the setting of neoadjuvant chemotherapy. 
45. Vuppalanchi R, Chalasani N. Nonalcoholic fatty liver disease and nonalcoholic steatohepatitis: selected practical issues in their evaluation and management. Hepatology. 2009;49:306-17.

46. Peppercorn PD, Reznek RH, Wilson P, Slevin ML, Gupta RK. Demonstration of hepatic steatosis by computerized tomography in patients receiving 5-fluorouracil-based therapy for advanced colorectal cancer. Br J Cancer. 1998;77:2008-11.

47. Davidson LE, Kuk JL, Church TS, Ross R. Protocol for measurement of liver fat by computed tomography. J Appl Physiol. 1985;2006(100):864-8.

48. - Noworolski SM, Lam MM, Merriman RB, Ferrell L, Qayyum A. Liver steatosis: concordance of MR imaging and MR spectroscopic data with histologic grade. Radiology. 2012;264:88-96. Evaluation of MR imaging in the setting of steatosis.
49. Kulemann V, Schima W, Tamandl D, et al. Preoperative detection of colorectal liver metastases in fatty liver: MDCT or MRI? Eur J Radiol. 2011;79:e1-6.

50. Berger-Kulemann V, Schima W, Baroud S, et al. Gadoxetic acidenhanced 3.0 T MR imaging versus multidetector-row CT in the detection of colorectal metastases in fatty liver using intraoperative ultrasound and histopathology as a standard of reference. Eur J Surg Oncol. 2012;38:670-6.

51. • van Vledder MG, Torbenson MS, Pawlik TM, et al. The effect of steatosis on echogenicity of colorectal liver metastases on intraoperative ultrasonography. Arch Surg. 2010;145:661-67. This report demonstrates that IOUS continues to play a major role in the treatment of CRC with liver metastases even in the setting of hepatic steatosis. 